\title{
Micro-computed tomography evaluation of general trends in aligner thickness and gap width after thermoforming procedures involving six commercial clear aligners: An in vitro study
}

\author{
Mario Palone ${ }^{\mathrm{a}}$ (1) \\ Mattia Longo $^{\mathrm{a}}$ \\ Niki Arveda ${ }^{\mathrm{a}}$ \\ Michele Nacucchi ${ }^{\mathrm{b}}$ \\ Fabio De Pascalis ${ }^{b}$ \\ Giorgio Alfredo Spedicato ${ }^{c}$ \\ Giuseppe Siciliani $^{\mathrm{d}}$ \\ Luca Lombardo ${ }^{\mathrm{a}}$ (10
}

Postgraduate School of Orthodontics, University of Ferrara, Ferrara, Italy

${ }^{\mathrm{b}}$ Division for Sustainable Materials, Research Centre of Brindisi, Brindisi, Italy

'School of Economics, Management and Statistics, University of Bologna, Bologna, ltaly

${ }^{\mathrm{d}}$ Department of Translational Medicine and for Romagna, School of Dentistry, University of Ferrara, Ferrara, Italy
Objective: To assess the effects of thermoforming on aligner thickness and gap width in six aligner systems with the same nominal thickness. Methods: Six passive upper aligners of different brands were adapted to a single printed cast. Each sample was evaluated with high-resolution micro-computed tomography. To investigate aligner thickness and gap width, two-dimensional (2D) analysis was conducted assessing the effects of the following variables: tooth type (central incisor, canine, and first molar), 2D reference points, and aligner type. Data were analyzed and compared using analysis of variance and Tukey's post-hoc tests $(p<0.05)$. Results: Tooth type, dental region, and aligner type affected both the gap width and aligner thickness. The aligner thickness remained moderately stable across the arch only in the F22. Conclusions: All thermoformed samples displayed smaller aligner thickness and gap width at anterior teeth and both gingival and coronal centers than at posterior teeth and occlusal surfaces.

[Korean J Orthod 2021;51(2):135-141]

Key words: Gap width, Aligner thickness, Thermoforming procedure, Clear aligner therapy

Received May 13, 2020; Revised November 28, 2020; Accepted December 9, 2020.

Corresponding author: Mario Palone.

Research Fellow, Postgraduate School of Orthodontics, University of Ferrara, Via Luigi Borsari 46, Ferrara 44121, ltaly.

Tel +39-3289523202 e-mail mario.palone88@gmail.com

Giorgio Alfredo Spedicato's current affiliation: Department of Banking and Insurance, Catholic University of Milan, Milano, Italy.

How to cite this article: Palone M, Longo M, Arveda N, Nacucchi M, De Pascalis F, Spedicato GA, Siciliani G, Lombardo L. Micro-computed tomography evaluation of general trends in aligner thickness and gap width after thermoforming procedures involving six commercial clear aligners: An in vitro study. Korean J Orthod 2021;51:135-141.

(C) 2021 The Korean Association of Orthodontists.

This is an Open Access article distributed under the terms of the Creative Commons Attribution Non-Commercial License (http://creativecommons.org/licenses/by-nc/4.0) which permits unrestricted non-commercial use, distribution, and reproduction in any medium, provided the original work is properly cited. 


\section{INTRODUCTION}

Despite the widespread use of aligners, several factors, including their thickness and fit (i.e., gap width), have been scarcely investigated, even though these factors are closely connected to other important features such as their optical properties, ${ }^{1,2}$ the forces and moments they generate ${ }^{3,4}$ their retention, ${ }^{5}$ and the predictability of the prescribed movements. ${ }^{6}$ There is even less information available on the physical and chemical properties of the thermoplastic materials used to make aligners once they are thermoformed. However, orthodontists need to be aware of the mechanical and chemical properties of aligner materials after the thermoforming process, ${ }^{2}$ since the process significantly influences the mechanical and chemical properties of the aligners themselves. ${ }^{7-9}$

Although recent research has focused on analysis of three-dimensional (3D) printed aligners, ${ }^{10}$ thermoforming is the method most commonly used to manufacture aligners. ${ }^{11}$ In thermoformed aligners, the fit depends on the manufacturing processes (set temperature and pressure), the elastic modulus of the materials used, ${ }^{12}$ the presence of divots and attachments, ${ }^{13}$ and the hygroscopic expansion phenomena when the aligner is in contact with saliva or water. ${ }^{14}$ Mantovani et al..$^{12,13}$ investigated the fit of various aligner systems using microphotographs obtained by scanning electron microscopy of buccolingual sections of passive aligners obtained by a cutting machine and adapted to a 3D printed cast. Considering the risk, albeit minimal, of distortion and/or roughening of the cut edges, Lombardo et al. ${ }^{15}$ investigated aligner thickness and gap width using noninvasive two-dimensional (2D) and 3D analysis performed by micro-computed tomography (CT), and they found heterogeneity among the aligner samples investigated.

Aligner thickness of the occlusal surfaces has also been analyzed using a 3D technique in both as-received and as-retrieved aligners (after 10 days in the oral cavity) in both passive and active configurations, demonstrating good stability. Although interesting, that analysis was limited to occlusal surfaces and aligners made of one material, glycol modified polyethylene terephthalate, via one thermoforming protocol, ${ }^{11}$ but there are numerous aligner systems on the market. ${ }^{16}$

Thus, the aim of this study was to evaluate and compare the general effects of thermoforming processes on both gap width and thickness via micro-CT investigation of passive aligners with the same nominal thickness obtained from six manufacturers.

The informations about thermoforming processes could be useful to clinicians, who have the intention to set their staging protocol during clear aligner therapy (CAT) without overloading the periodontal ligament and good efficiency.

\section{MATERIALS AND METHODS}

The design of this in vitro study was approved by the University of Ferrara, Postgraduate School of Orthodontics Ethics Committee with protocol number 8-2017. Polyvinyl siloxane (PVS) impressions (Elite HD+ Regular and Light Body; Zhermack, Badia Polesine, Italy) were obtained from one patient with skeletal Class 1 and slight crowding and misalignment in both arches who had requested esthetic treatment. The patient had no conservative, endodontic, or prosthetic issues, missing teeth, reduced clinical crowns, or coronal surface abnormalities.

The PVS impressions, together with the patient's records, were sent to Align Technology (Santa Clara, CA, USA), who accept only PVS impressions or STL files generated by an 1-Tero ${ }^{\circledR}$ Element ${ }^{\mathrm{TM}}$ scanner (Align Technology). During processing, the STL file was obtained via the Clin Check Pro ${ }^{\circledR}$ website (Align Technology), and this, together with complete patient records, was sent to the manufacturers of six aligners (Airnivol, ALL IN, Arc Angel, F22, Invisalign, and Nuvola) listed in Table 1. Each manufacturer was apprised of the same treatment objectives by a single expert aligner clinician. After approving the proposed treatment plan, the same operator

Table 1. List of the six commercial aligner systems investigated, with their respective thicknesses and construction materials

\begin{tabular}{lclcl}
\hline Aligner & Specimen & Material & $\begin{array}{c}\text { Thickness } \\
(\mathbf{m m})\end{array}$ & \multicolumn{1}{c}{ Manufacturer } \\
\hline Airnivol & 1 & Polyethylene terephtalate glycol (PET-G) & 0.75 & Airnivol srl, Navacchio di Cascina, PI, Italy \\
ALL IN & 1 & Polyethylene terephtalate glycol (PET-G) & 0.80 & Micerium, Avegno, GE, Italy \\
Arc Angel & 1 & Polyethylene terephtalate glycol (PET-G) & 0.75 & Gruppo Dextra, Modena, MO, Italy \\
F22 & 1 & F22 Polyurethane & 0.75 & Sweden-Martina, Due Carrare, PD, Italy \\
Invisalign & 1 & $\begin{array}{l}\text { SmartTrack: multi-layer aromatic } \\
\text { thermoplastic polyurethane }\end{array}$ & 0.75 & Align Technology, Santa Clara, CA, USA \\
Nuvola & 1 & Polyethylene terephtalate glycol (PET-G) & 0.75 & GEO srl, Rome, RM, Italy \\
\hline
\end{tabular}


explicitly requested a single fully passive aligner.

The patient's STL file was used to 3D-print a single upper cast (E-Dentstone M; EnvisionTEC, Gladbeck, Germany) using an ULTRA 3SP Ortho 3D Dental Printer (EnvisionTEC); after calibration of the 3D printer, the definition was set at $50 \mu \mathrm{m}$ to minimize distortion error. A single 3D cast, rather than one per manufacturer, was used to reduce the effect of any potential micrometric variations. The model was washed, and each of the six sample aligners was mounted in turn for microtomography using nano-CT GE Phoenix Nanotom (GE Sensing Et Inspection Technologies GmbH, Wunstorf, Germany), performed at the ENEA Research Centre (Brindisi, Italy), as in a previous study. ${ }^{15}$ Each aligner was left in place until microtomography had been completed in order to prevent possible distortion.

\section{D analysis}

To evaluate the aligner thickness and the volume of air (gap width) between the aligner and cast, tomographic microphotographs of three virtual slices corresponding to the three tooth types investigated (central incisor, canine, and first molar), yielding a total of 18 slices, were obtained for each sample analyzed. The mechanism of slice plan orientation is the same as that used in a previous study. ${ }^{15}$ Subsequently, ImageJ software (NIH ImageJ Software, https://imagej.nih.gov/ij/), an open-source scientific image analysis package, was used to construct grids on the microphotographs thereby obtained, at a relative magnification of 3,200x, for each tooth, i.e., 3 grids per sample. On each 2D grid, several reference points were identified:

- 5 for the central incisor
- 5 for the canine

- 8 for the first molar (Figure 1).

Both measurements were obtained by tracing a line perpendicular to the tangent of the tooth, passing through the reference point on the grid. After 4 weeks, 92 measurements, equal to $45 \%$ of the sample, were repeated, and the method error was calculated according to Dahlberg's formula $\left(S^{2}=\sum d^{2} / 2 n\right) .{ }^{17}$ The systematic error was measured using Student's $t$-test, with a $p$ value of $<0.05$ considered significant. The replicability appeared to be good, since a (paired) sample $t$-test on 92 paired measures showed a t-statistic equal to 0.38 and a corresponding $p$-value equal to 0.7. The Dahlberg statistic was $0 \mathrm{~mm}$, numerically close to zero.

\section{Statistical analysis}

The data obtained from the 204 2D linear measurements on tomographic microphotographs at a relative enlargement of 3,200x were statistically tested using one-way analysis of variance (ANOVA) by R Statistical software (R Core Team 2018). ${ }^{18}$ This statistical analysis enabled us to investigate the behavior of both the aligner thickness and gap width based on the variables tooth type (central incisor, canine or first molar), 2D reference point, and aligner type on the tomographic microphotograph grid. Statistical significance was assessed using a 5\% threshold. A significant $p$-value indicates that at least one group is different from the overall mean.

All analyses were split by measure. Tukey's post-hoc comparison of means indicated which pair of measures were statistically different.

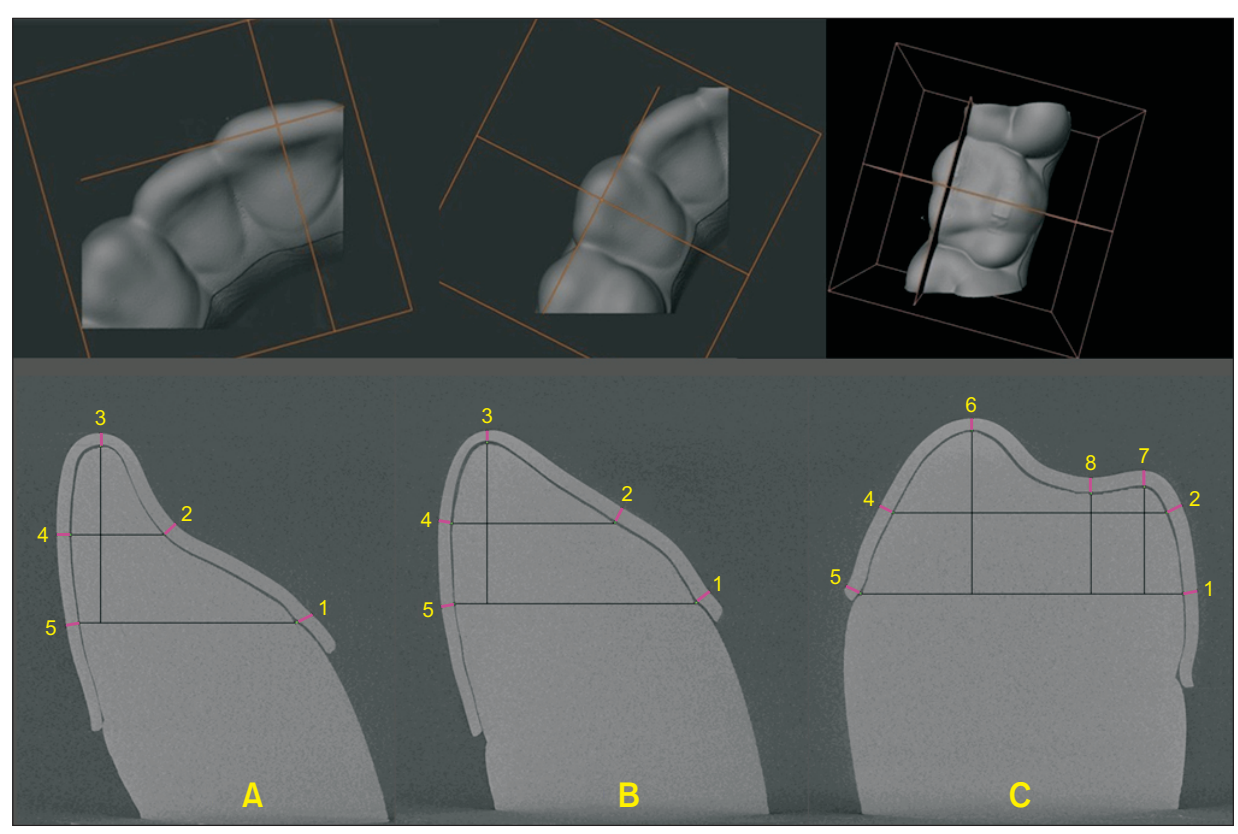

Figure 1. Identification of slice planes and two-dimensional reference points on the construction grid for the incisor $(A)$, canine $(B)$, and first molar (C). Eight points were identified: 1, palatal gingival edge; 2 , palatal surface center; 3 , incisal edge; 4 , vestibular surface center; 5 , vestibular gingival edge; 6 , vestibular cusp; 7, palatal cusp; and 8, central groove. 


\section{RESULTS}

For the tooth type variable, one-way ANOVA revealed statistically significant differences in both aligner thickness $(\mathrm{F}=9.68, p=0)$ and gap width $(\mathrm{F}=3.82, p=$ 0.025) (Table 2). In a comparison of the gap width at the first molar with those at the incisor and canine, there was a difference of roughly $0.1 \mathrm{~mm}$, with a bor- derline statistical significance $(p=0.06)$. Analysis of the aligner thickness, on the other hand, showed significant differences between the first molar and those at the other teeth investigated $(p<0.05)$. However, no significant differences between incisor and canine were detected in either measurements (Table 3).

One-way ANOVA of 2D reference point data revealed statistically significant differences in both aligner thick-

Table 2. Mean gap width and aligner thickness measurements $(\mathrm{mm})$ with their respective standard deviations $(\mathrm{mm})$

\begin{tabular}{llcccccccc}
\hline Measurement & Tooth & Number & $\begin{array}{c}\text { Mean } \\
(\mathbf{m m})\end{array}$ & $\begin{array}{c}\text { Standard } \\
\text { deviation } \\
(\mathbf{m m})\end{array}$ & $\begin{array}{c}\text { Tooth } \\
\text { comparison }\end{array}$ & $\begin{array}{c}\text { Estimate } \\
(\mathbf{m m})\end{array}$ & $\begin{array}{c}\text { Lower } \\
\mathbf{C L} \\
(\mathbf{m m})\end{array}$ & $\begin{array}{c}\text { Upper } \\
(\mathbf{m m})\end{array}$ & p-value \\
\hline Gap width & Canine & 30 & 0.141 & 0.086 & Canine-Incisor & 0 & -0.11 & 0.11 & 1 \\
& Incisor & 30 & 0.141 & 0.110 & First Molar-Canine & 0.1 & 0 & 0.2 & 0.06 \\
& First molar & 42 & 0.239 & 0.244 & First Molar-Incisor & 0.1 & 0 & 0.2 & 0.06 \\
Aligner thickness & Canine & 30 & 0.520 & 0.091 & Canine-Incisor & 0.01 & -0.04 & 0.06 & 0.94 \\
& Incisor & 30 & 0.512 & 0.067 & First Molar-Incisor & 0.08 & 0.03 & 0.13 & $0^{*}$ \\
& First molar & 42 & 0.590 & 0.085 & First Molar-Canine & 0.07 & 0.02 & 0.12 & $0^{*}$ \\
\hline
\end{tabular}

CL, confidence limit.

Tukey's post-hoc comparisons of mean gap width and aligner thickness values among the different teeth are performed $\left({ }^{*} p<\right.$ 0.05).

Statistical comparison of mean gap width and aligner thickness values among the different teeth analyzed $\left({ }^{*} p<0.05\right)$.

Table 3. Mean linear values $(\mathrm{mm})$ and standard deviations $(\mathrm{mm})$ for gap width and aligner thickness at each 2D reference points (see Figure 1) and their respective comparisons

\begin{tabular}{lccccccccc}
\hline Measurement & $\begin{array}{c}\text { 2D } \\
\text { points }\end{array}$ & Number & $\begin{array}{c}\text { Mean } \\
(\mathbf{m m})\end{array}$ & $\begin{array}{c}\text { Standard } \\
\text { deviation } \\
(\mathbf{m m})\end{array}$ & $\begin{array}{c}\text { 2D } \\
\text { point } \\
\text { comparisons }\end{array}$ & Diff & $\begin{array}{c}\text { Lower } \\
\text { CL } \\
(\mathbf{m m})\end{array}$ & $\begin{array}{c}\text { Upper } \\
\text { CL } \\
(\mathbf{m m})\end{array}$ & p-value \\
\hline Gap width & 1 & 18 & 0.183 & 0.111 & & & & & \\
& 2 & 18 & 0.149 & 0.078 & $6-5$ & 0.240 & 0.014 & 0.466 & $0.029^{*}$ \\
& 3 & 12 & 0.200 & 0.106 & $7-5$ & 0.268 & 0.042 & 0.494 & $0.009^{*}$ \\
& 4 & 18 & 0.109 & 0.091 & $8-5$ & 0.329 & 0.103 & 0.554 & $0.000^{*}$ \\
& 5 & 18 & 0.085 & 0.063 & $7-4$ & 0.244 & 0.018 & 0.470 & $0.025^{*}$ \\
& 6 & 6 & 0.324 & 0.227 & $8-4$ & 0.304 & 0.079 & 0.530 & $0.002^{*}$ \\
& 7 & 6 & 0.353 & 0.338 & $8-2$ & 0.264 & 0.038 & 0.490 & $0.011^{*}$ \\
& 8 & 6 & 0.413 & 0.377 & & & & & \\
& 1 & 18 & 0.548 & 0.048 & $8-3$ & 0.132 & 0.008 & 0.255 & $0.029^{*}$ \\
& 1 & 18 & 0.563 & 0.043 & & & & & \\
& 2 & 12 & 0.493 & 0.106 & $6-3$ & 0.170 & 0.046 & 0.294 & $0.001^{*}$ \\
& 3 & 18 & 0.549 & 0.106 & & & & & \\
& 4 & 18 & 0.500 & 0.084 & $8-5$ & 0.125 & 0.008 & 0.242 & $0.027^{*}$ \\
& 5 & 6 & 0.663 & 0.067 & & & & & \\
& 6 & 6 & 0.522 & 0.078 & $6-5$ & 0.163 & 0.047 & 0.280 & $0.001^{*}$ \\
& 7 & 6 & 0.625 & 0.061 & & & & & \\
\hline
\end{tabular}

Each measurement is derived from a single passive aligner for each brand investigated.

2D, two-dimensional; CL, confidence limit.

Tukey's post-hoc comparisons that reached statistical significance are listed in the table $\left({ }^{*} p<0.05\right)$. 


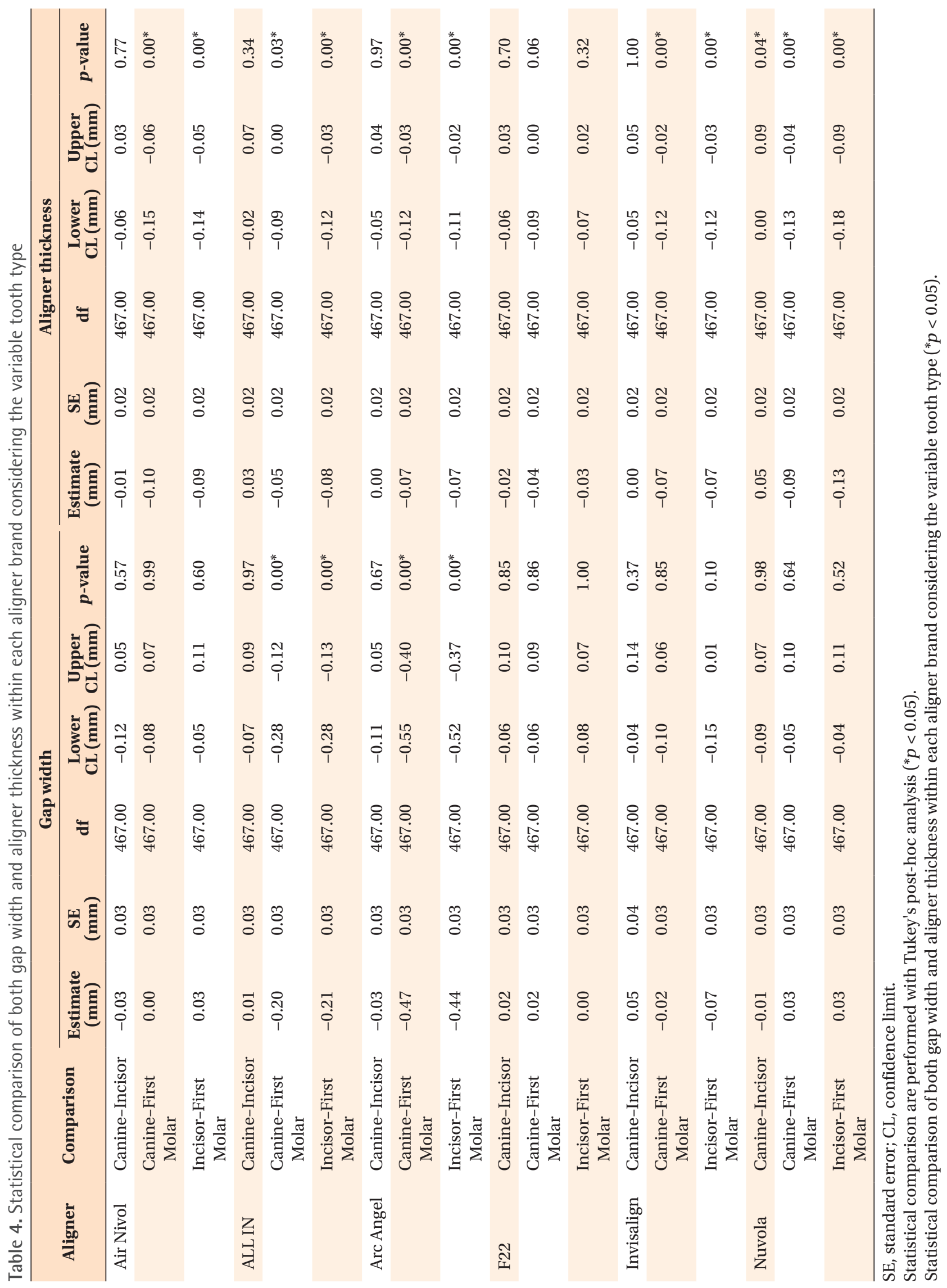


ness $(\mathrm{F}=4.51, p=0)$ and gap width $(\mathrm{F}=5.44, p=$ $0)$. Six differences in gap width and four differences in aligner thickness were significant (Table 3). Withingroup comparisons revealed significant differences in gap width $(p<0.05)$ between the anterior and posterior sectors, but only for the Arc Angel and ALL IN aligners. In contrast, statistically significant differences were observed in aligner thickness at the anterior and posterior teeth in all aligners except for the F22, which displayed greater homogeneity from front to back (Table 4).

\section{DISCUSSION}

The aim of this study was to clarify how dental anatomy can influence gap width and aligner thickness after thermoforming procedures. In assessments of the gap between the aligner and teeth, our results reveal differences of about $0.1 \mathrm{~mm}$ between the anterior (incisor and canine) and posterior regions, albeit of borderline statistical significance. Our 2D analysis also showed that the fit is generally better at the gingival as opposed to the occlusal regions of the first molar. These aspects appear to be due to greater stretching of the plastic material at the gingival areas and at the anterior tapered teeth during the thermoforming process. These data are in line with those reported by Mantovani et al. ${ }^{12}$ and are important to note because the fit between the internal surface of the aligner and the tooth surface seems to influence orthodontic force transmission by active aligners and the onset of tooth movement, ${ }^{15}$ as well the capacity of passive retainers to prevent relapse. Indeed, the contact between aligner and tooth surfaces needs to be as close as possible to achieve clinically efficacious forces from the initial stages of aligner therapy considering both the air in the gap width and the volume occupied by the ligament, which ensures about $0.04 \mathrm{~mm}$ of free tooth movement before any chemical change can be detected. $^{19}$

Considering these aspects, the molar sectors showed mean gap widths that exceeded the staging protocols common to many aligner systems $(0.25-0.33 \mathrm{~mm})$, especially at the occlusal surfaces. Orthodontists should take this into account when planning orthodontic movement, especially posterior intrusion. For passive aligners, on the other hand, a minimum gap width is linked to the capacity to counteract both vertical dislodging forces and orthodontic relapse.

With regard to the modification of nominal aligner thickness by thermoforming procedures which could affect the orthodontic forces transmitted, our study showed that that aligners were generally thinner at the anterior and occlusal regions, which was in agreement with that of Bucci et al. ${ }^{11}$ The greater thickness at the posterior occlusal surfaces could facilitate vertical control of the posterior teeth during CAT in the treatment of dental open bite. ${ }^{20,21}$ Our comparative analysis revealed a diminishing trend in thickness from posterior to anterior teeth common to all aligners investigated, with the exception of F22 aligners, whose thickness was constant across the arch. Although this difference may be clinically insignificant, orthodontists should bear in mind the difference in aligner thickness between the anterior and posterior sectors, increasing the movement staging at anterior teeth without overloading the periodontal tissue. For passive aligners, their thickness is directly linked to their stiffness, which could prevent undesired orthodontic movements during the retention period as well as posterior vertical stability in high angle subjects; on the other hand, a subsequent major gap width should be considered based on the results of this study.

In summary, all systems fit better at anterior than at posterior teeth, but the difference reached statistical significance only for the ALL IN and Arc Angel aligners. We also observed decreased aligner thickness toward the anterior regions (except for F22) and at gingival sites with respect to the occlusal regions, which could influence the amount of force expressed. Larger differences were found in terms of aligner fit, since the gap tended to be smaller at the anterior sector than the posterior. The greatest difference detected was roughly $0.1 \mathrm{~mm}$, which may be sufficient to affect the onset and predictability of orthodontic movement in the posterior sectors. However, the small size of the differences, even those that were statistically significant, makes them difficult to quantify. Furthermore, since this was in vitro study, it did not take into account several factors that could reduce the thickness and/or fit of aligners (saliva and occlusal forces). Moreover, divots, attachments, or grip points were not taken into consideration. In addition, a single specimen of each aligner brand was tested, so the results may not be representative, and could be partially negatively influenced by undesired temperature and pressure settings. This should therefore be considered a pilot study. In future investigations, multiple aligners of each brand and several other factors should be taken into consideration to evaluate the reproducibility of thermoforming procedures.

\section{CONCLUSION}

- The nominal aligner thickness was always reduced by thermoforming.

- Aligners were generally thinner and the aligner gap narrower at the anterior teeth and gingival regions. Aligners were thicker and the aligner gap wider at the posterior teeth and occlusal surfaces.

- The diminishing aligner thickness trend from pos- 
terior to anterior sectors was common to all aligners investigated, with the exception of F22, which showed a more homogeneous trend.

- Clinicians should keep into account these differences across the arch, in order to choose the most suitable staging protocol for teeth movements. Authors encourage manufactures to make accessible to clinicians these data.

\section{CONFLICTS OF INTEREST}

No potential conflict of interest relevant to this article was reported.

\section{REFERENCES}

1. Lombardo L, Arreghini A, Maccarrone R, Bianchi A, Scalia S, Siciliani G. Optical properties of orthodontic aligners--spectrophotometry analysis of three types before and after aging. Prog Orthod 2015;16:41.

2. Ryu JH, Kwon JS, Jiang HB, Cha JY, Kim KM. Effects of thermoforming on the physical and mechanical properties of thermoplastic materials for transparent orthodontic aligners. Korean J Orthod 2018;48:316-25.

3. Gao L, Wichelhaus A. Forces and moments delivered by the PET-G aligner to a maxillary central incisor for palatal tipping and intrusion. Angle Orthod 2017;87:534-41.

4. Elkholy F, Schmidt F, Jäger R, Lapatki BG. Forces and moments applied during derotation of a maxillary central incisor with thinner aligners: an invitro study. Am J Orthod Dentofacial Orthop 2017;151:407-15.

5. Cowley DP, Mah J, O'Toole B. The effect of gingival-margin design on the retention of thermoformed aligners. J Clin Orthod 2012;46:697-702; quiz 705.

6. Lombardo L, Arreghini A, Ramina F, Huanca Ghislanzoni LT, Siciliani G. Predictability of orthodontic movement with orthodontic aligners: a retrospective study. Prog Orthod 2017;18:35.

7. Lombardo L, Toni G, Stefanoni F, Mollica F, Guarneri MP, Siciliani G. The effect of temperature on the mechanical behavior of nickel-titanium orthodontic initial archwires. Angle Orthod 2013;83:298-305.

8. Martina S, Rongo R, Bucci R, Razionale AV, Valletta $R$, D'Antò V. In vitro cytotoxicity of different thermoplastic materials for clear aligners. Angle Orthod
2019;89:942-5.

9. Alexandropoulos A, Al Jabbari YS, Zinelis S, Eliades T. Chemical and mechanical characteristics of contemporary thermoplastic orthodontic materials. Aust Orthod J 2015;31:165-70.

10. Jindal P, Juneja M, Siena FL, Bajaj D, Breedon P. Mechanical and geometric properties of thermoformed and 3D printed clear dental aligners. Am J Orthod Dentofacial Orthop 2019;156:694-701.

11. Bucci R, Rongo R, Levatè C, Michelotti A, Barone S, Razionale AV, et al. Thickness of orthodontic clear aligners after thermoforming and after 10 days of intraoral exposure: a prospective clinical study. Prog Orthod 2019;20:36.

12. Mantovani E, Castroflorio E, Rossini G, Garino F, Cugliari G, Deregibus A, et al. Scanning electron microscopy evaluation of aligner fit on teeth. Angle Orthod 2018;88:596-601.

13. Mantovani E, Castroflorio E, Rossini G, Garino F, Cugliari G, Deregibus A, et al. Scanning electron microscopy analysis of aligner fitting on anchorage attachments. J Orofac Orthop 2019;80:79-87.

14. Ryokawa H, Miyazaki Y, Fujishima A, Miyazaki T, Maki K. The mechanical properties of dental thermoplastic materials in a simulated intraoral environment. Orthod Waves 2006;65:64-72.

15. Lombardo L, Palone M, Longo M, Arveda N, Nacucchi M, De Pascalis F, et al. MicroCT X-ray comparison of aligner gap and thickness of six brands of aligners: an in-vitro study. Prog Orthod 2020;21:12.

16. Gierie WV. Clear aligner therapy: an overview. J Clin Orthod 2018;52:665-74.

17. Dahlberg G. Statistical methods for medical and biological students. London: Interscience Publishers Inc.; 1940.

18. R Development Core Team RR. A language and environment for statistical computing [Internet]. R Development Core Team RR; 2011 [cited 2020 Feb 29]. Available from: http://www.r-project.org.

19. Dorow C, Krtsin N, Sander FG. In vivo experimental investigation of tooth mobility in humans. Biomed Tech 2002;47:20-5.

20. Moshiri S, Araújo EA, McCray JF, Thiesen G, Kim KB. Cephalometric evaluation of adult anterior open bite non-extraction treatment with Invisalign. Dental Press J Orthod 2017;22:30-8.

21. Ojima K, Dan C, Watanabe H, Kumagai Y, Nanda R. The biomechanics of aligner orthodontics in openbite cases. J Clin Orthod 2019;53:699-712. 